\title{
Calculation of the protective current density distribution of a cathodic protection system with galvanic anodes in terms of double-layer electrolyte
}

\author{
A. Mujezinović ${ }^{1}$, Ai. Muharemović ${ }^{2}$, I. Turković ${ }^{1} \&$ \\ Ad. Muharemović ${ }^{3}$ \\ ${ }^{I}$ Faculty of Electrical Engineering, University of Sarajevo, \\ Bosnia \& Herzegovina \\ ${ }^{2}$ BH Telecom d.d. Sarajevo, Bosnia \& Herzegovina \\ ${ }^{3}$ Independent System Operator, Sarajevo, Bosnia \& Herzegovina
}

\begin{abstract}
This paper considers the effect of discontinuity of electrical conductivity of electrolytes on the distribution of potential and protective current density in cathodic protection systems with galvanic anodes. The aim of the paper is to present a simultaneous analysis for application of both analytical and numerical models for calculation of the distribution of protective potential and current density in the cases of homogeneous and double-layer electrolytes. When considering non-linear boundary conditions at the electrode surface (secondary distribution of protective current density), the indirect boundary element method is used, because of the complexity of the calculation. Collocation at the point method was used for calculation. Due to the nonlinearity of boundary conditions at the electrode surfaces, the calculation problem is further complicated. In order to show the importance of this analysis, calculations for the observed system as well as calculations of errors caused by neglecting the boundary discontinuity of the soil conductivity are provided. Based on calculations and with respect to error analyses, the conclusive remark gives the impact valuation of the doublelayer electrolyte on the correct calculation of the cathodic protection system with galvanic anodes. This paper also gives the analysis when the double-layered nature of the electrolyte can practically be ignored, which has great importance for designers of these systems.
\end{abstract}


Keywords: cathodic protection (CP), distribution of the protection current density, single-layer electrolyte, double-layer electrolyte.

\section{Introduction}

Cathodic protection (CP) is a technique that prevents corrosion of underground metallic structures. Factors that may affect the parameters of the modern $\mathrm{CP}$ systems are indispensable data for project documentation. They present a multidisciplinary phenomenon that complicates the definition of CP system parameters. The potential distribution of anode strings is determined for each specific case and it depends on the real polarization curves. The polarization curves, in the mathematical sense, define the boundary conditions for determining the value of the electrode potential. The BEM method used for calculation of the protective potential and the current density of long pipelines, with either larger or smaller insulation damage, is well covered in the paper of Riemer and Orazema [1]. Riemer has developed the calculation method that takes into account the potential drop in the pipeline, i.e. attenuation that is not balanced over the entire pipeline [2]. Adey et al. have incorporated a full 3D model for calculating the potential of the CP system, also including neighboring objects that have connections to the anodes [3]. Peratta et al. have applied the ML-BEM method for calculation of the parameters of the CP system in multilayer soil [4].

Calculations of the distribution of both the potential and protective current density present the base of the CP system project. Factors that complicate the calculation are non-linear boundary conditions on electrode surfaces. In addition, the calculation needs to take into account the occurrence of any boundary discontinuity of electrical soil conductivity. The electrolyte, in which the metallic pipeline is buried, is mostly inhomogeneous (layered) and has two or more layers of different values of electrolyte conductivity [5]. Based on what is mentioned above, it becomes clear that the analytical solution of this problem is only partial and superficial.

\section{Calculation of distribution of protective current density in cathodic protection systems with galvanic anodes}

This paper indicates that there is a substantial difference between methods used for determining the parameters of the CP system, as well as in the treatment of boundary conditions:

- The analytical method that treats the primary distribution of the protective current density, taking into account the characteristics of the electrolyte and the geometry of the system $[6,7]$, and

- The numerical method that treats the secondary distribution of the protective current density, i.e. taking into account the kinetic effects that are specific for the electrode surface, and that result in nonlinear boundary conditions $[8,9]$. 


\subsection{Primary distribution of the protective current density}

\subsubsection{Primary distribution of the protective current density of the \\ CP system with galvanic anodes in homogeneous electrolytes}

As previously mentioned, when modeling the primary distribution of the protective current density, only the electrical and geometrical characteristics of corrosive elements are relevant. In this section of the paper, we analyze an example of the galvanic anode placed in the homogeneous electrolyte. If the DC source is installed as the galvanic anode at some location in the electrolyte, with coordinates $\left(x^{\prime}, y^{\prime}, z^{\prime}\right)$, then the potential $\varphi$ at any point of the electrolyte with coordinates $P(x, y, z)$, at the distance $R$, can be defined as:

$$
\varphi(x, y, z)=\frac{1}{4 \pi \gamma_{U}} \int_{S^{\prime}} \frac{J(x, y, z)}{R} d S^{\prime},
$$

where: $\gamma_{U}$ - electric conductivity of the electrolyte,

$J(x, y, z)$ - surface density of the protective current,

$R$ - distance between the source and observation points,

$d S^{`}$ - integration path.

In order to symplify the mathematical model, the observed anode string, which is shown in Figure 1, is equivalented with one anode, having total length equal to the sum of the lengths of individual anodes. When solving the primary distribution of the protective current density of CP systems with galvanic anodes, it is necessary to add a set of equations that take into account the boundary conditions at the interface between anode strings and the electrolyte. The simplest case is the one that considers the potential at the complete outer surface of the anode string as a constant value (this condition can only be used in the primary distribution because of neglect of the polarization on the surfaces of the galvanic anodes). Similarly, the potential change in the electrolyte is constant as well. The boundary conditions can be written as:

$$
\begin{gathered}
\varphi=\text { const } \\
\frac{\partial \varphi}{\partial \vec{n}}=\text { const } \\
\vec{n} \times\left(\frac{\vec{J}_{U}}{\gamma_{U}}-\frac{\vec{J}_{A}}{\gamma_{A}}\right)=0 \\
\vec{n} \cdot\left(\vec{J}_{U}-\vec{J}_{A}\right)=0,
\end{gathered}
$$

where: $\vec{n}$ - normal vector on the boundary surfaces,

$\gamma_{A}-$ electrical conductivity of air,

$\gamma_{U}$ - electrical conductivity of electrolyte,

$\vec{J}_{A}$ - current density in air at the discontinuity boundary of the electrical conductivity,

$\vec{J}_{U}$ - current density in electrolyte at the discontinuity boundary of the electrical conductivity. 


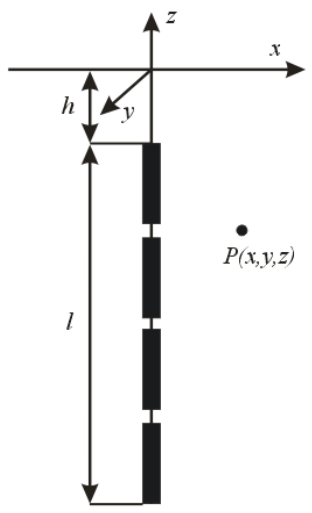

Figure 1: $\quad$ Anode string.

Because of its electrical conductivity $\left(10^{-18} \mathrm{~S} / \mathrm{m}\right)$, the air is treated as an insulator. Therefore, the mathematical analysis of the problem considers the mirror source at a distance $h$ away from the electrolyte/air boundary, with the same protective current density as the original, placed in the electrolyte (Figure 2.a). The expression for calculating the potential in the electrolyte, in the vicinity of the anodic string, is:

$\varphi(x, y, z)=\frac{I}{4 \pi l \gamma_{U}} \cdot\left(\ln \frac{z+l+h+\sqrt{(z+l+h)^{2}+r^{2}}}{z-l-h+\sqrt{(z-l-h)^{2}+r^{2}}}+\ln \frac{z-h+\sqrt{(z-h)^{2}+r^{2}}}{z+h+\sqrt{(z+h)^{2}+r^{2}}}\right)$

where: $l$ - total length of the equivalent anode string,

$h$ - depth of burying anode string,

$\gamma_{U}$ - electric conductivity of the single-layer electrolyte,

$I-$ total current intensity,

$$
r=x^{2}+y^{2}
$$

\subsubsection{Primary distribution of the protective current density of CP system with galvanic anodes in double-layer electrolytes}

Soil as the electrolyte is mainly composed of more layers, which differ in chemical and geological composition. When expecting a large difference in conductivity of the electrolyte layers, it is advisable to use a double-layer model of the electrolyte. In a case of simple geometry of electrodes, such as those considered in the paper, the easiest way to find a solution is by using a method of multiple imaging.

In order to estimate the exact scalar value of the electric potential of the equivalent anodic strings, it is necessary to determine the position of equivalent galvanic strings, as well as their current densities. Furthermore, it is necessary to 
set appropriate boundary conditions. The boundary conditions applicable in this situation are almost identical to the boundary conditions for a homogeneous electrolyte. The equivalent anode string as well as its images have constant potential, the potential change within each medium is constant and on all the boundaries between the media apply boundary conditions given by eqns. (2.c) and (2.d). The double-layer model considers high-order mirror images that have a direct impact on the potential of the equivalent anode (Figure 2.b). The impact of the high-order image attenuates as the distance between the galvanic string and image increases, i.e. as the order of the image increases. Therefore, when calculating the potential of the anode surface, it is good enough to consider second order images. The effects of mirror images can be simply calculated as a sum of effects of individual images. The easiest way of calculating the potential of the equivalent galvanic string, buried in the double-layer electrolyte, is by using the following relations:

$$
\varphi(x, y, z)=\frac{I}{4 \pi \cdot l \cdot \gamma_{U}}\left[f_{1}+\alpha \cdot f_{2}+\beta \cdot f_{3}+\alpha \cdot \beta \cdot\left(f_{4}+f_{5}\right)\right],
$$

where:

$$
\begin{gathered}
\alpha \approx 1 \\
\beta=\frac{\gamma_{U}-\gamma_{L}}{\gamma_{U}+\gamma_{L}} \\
f_{1}=\ln \frac{z+l+h+\sqrt{(z+l+h)^{2}+r^{2}}}{z-l-h+\sqrt{(z-l-h)^{2}+r^{2}}}
\end{gathered}
$$

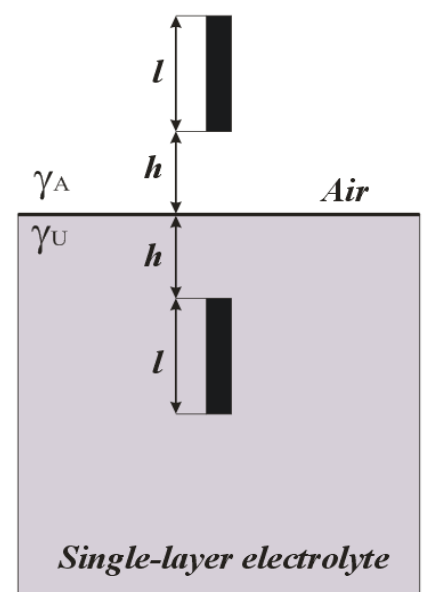

a)

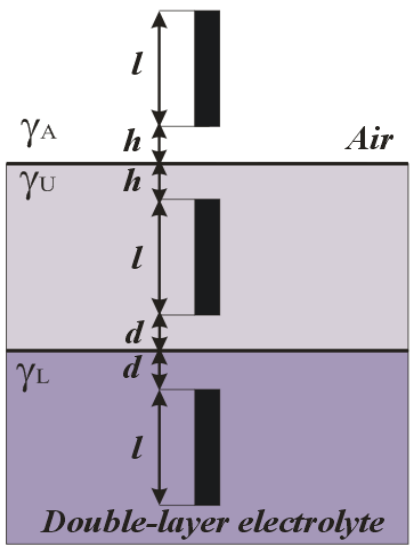

b)

Figure 2: Equivalent anode in a) single-layer electrolyte; b) double-layer electrolyte. 


$$
\begin{gathered}
f_{2}=\ln \frac{z-h+\sqrt{(z-h)^{2}+r^{2}}}{z+h+\sqrt{(z+h)^{2}+r^{2}}} \\
f_{3}=\ln \frac{z+2 l+h+2 d+\sqrt{(z+2 l+h+2 d)^{2}+r^{2}}}{z-2 l-h-2 d+\sqrt{(z-2 l-h-2 d)^{2}+r^{2}}} \\
f_{4}=\ln \frac{z-2 d-h-l+\sqrt{(z-2 d-h-l)^{2}+r^{2}}}{z+2 d+h+l+\sqrt{(z+2 d+h+l)^{2}+r^{2}}} \\
f_{5}=\ln \frac{z+3 h+3 l+2 d+\sqrt{(z+3 h+3 l+2 d)^{2}+r^{2}}}{z-3 h-3 l-2 d+\sqrt{(z-3 h-3 l-2 d)^{2}+r^{2}}},
\end{gathered}
$$

where: $\gamma_{L}-$ soil conductivity of lower layer,

$d$-distance between anode and lower layer of the electrolyte.

The calculation results of the potential distribution in the vicinity of the anode string are presented below, according to eqns. (3) and (5). The total length of the equivalent anode string is $6 \mathrm{~m}$, and it is buried in a single-layer of soil (Figure 3.a) with soil resistivity $\rho_{U}=50 \Omega \mathrm{m}$. In the second example (Figure 3.b) of the anode string, with the same characteristics, is buried in the upper layer of doublelayer soil. The soil resistivity of the upper layer is $\rho_{U}=50 \Omega \mathrm{m}$ and that of the lower layer $\rho_{L}=1000 \Omega \mathrm{m}$. The total thickness of the upper layer is $10 \mathrm{~m}$ and the depth of anode string burial is $2.8 \mathrm{~m}$. When modeling this example, second order images are taken into account.

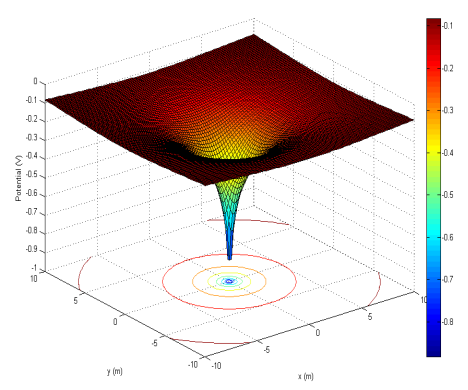

a)

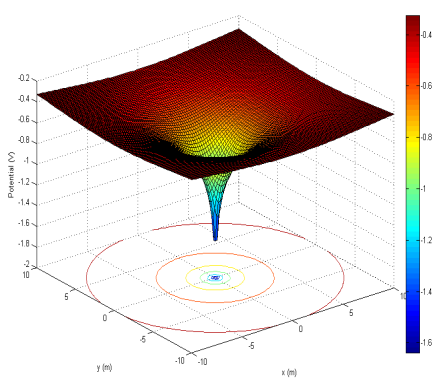

b)

Figure 3: Diagram of the potential distribution of the anode string in a) single-layer soil and b) double-layer soil. 


\subsection{Secondary distribution of protective current density}

When calculating the secondary distribution of the protective current density the kinetics of the electrode processes must be taken into account. Processes of hydrogen separation, dissolving of metal and oxygen reduction, simultaneously occur on the cathode surface. As a result of electrochemical reactions, the expression for the polarization current density on the cathode surface can be written as:

$$
j_{C}=j_{0, F e} \cdot 10^{\frac{\varphi-\varphi_{F e}}{\beta_{F e}}}-j_{\text {lim }, O_{2}} \cdot\left(1+10^{\frac{\varphi-\varphi_{O_{2}}}{\beta_{O_{2}}}}\right)^{-1}-j_{0, H_{2}} \cdot 10^{\frac{-\left(\varphi-\varphi_{H_{2}}\right)}{\beta_{H_{2}}}},
$$

where: $j_{C}$ - the sum of all partial current densities for cathode surface (bare steel); $j_{0, F e}$ - current density corresponding to the metal dissolution reaction; $j_{\text {lim } \mathrm{O}_{2}}-$ threshold current density of oxygen reduction; $j_{0, H_{2}}-$ current density corresponding to the reaction of hydrogen separation; $\varphi_{\mathrm{Fe}}, \varphi_{\mathrm{O}_{2}}, \varphi_{\mathrm{H}_{2}}-$ corrosion potentials for corresponding reactions; $\beta_{\mathrm{Fe}}, \beta_{\mathrm{O}_{2}}, \beta_{\mathrm{H}_{2}}-$ Tafel's coefficients (slopes); $\varphi$ - potential difference of interface metal/electrolyte.

Galvanic anodes are made from materials that are more electrically active than the cathode materials. Therefore, the dominant reaction on the anode surface is oxygen reduction. Mathematically, this can be defined as follows:

$$
j_{A}=j_{l i m, O_{2}} \cdot\left(10^{\frac{\varphi-\varphi_{\text {cor }, Z n}}{\beta_{Z n}}}-1\right) \text {, }
$$

where: $j_{A}-$ the sum current density for zinc anode,

$\varphi_{c o r, Z n}-$ natural potential of zinc,

$\beta_{Z n}-$ Tafel's coefficient (slope) for zinc.

The diagram in Figure 4 presents the boundary conditions on the electrode surfaces of the CP system with galvanic anodes. It is noticeable that these are highly nonlinear boundary conditions, especially on a cathode surface. The values of the protective potential of the cathode surface depend on the operating time of the $\mathrm{CP}$ system. At the beginning, i.e. at the time of $\mathrm{CP}$ commissioning, the cathode and anode surfaces get on the potential defined by points A and B, respectively. In this situation, the voltage drop in the electrolyte is defined by $j_{l} \cdot r_{p l \Sigma}$. After some time, i.e. after polarization of steel and the increase of the resistance of the anodic string, the potentials of the cathode and anode are defined by $A_{1}$ and $B_{1}$, respectively. 


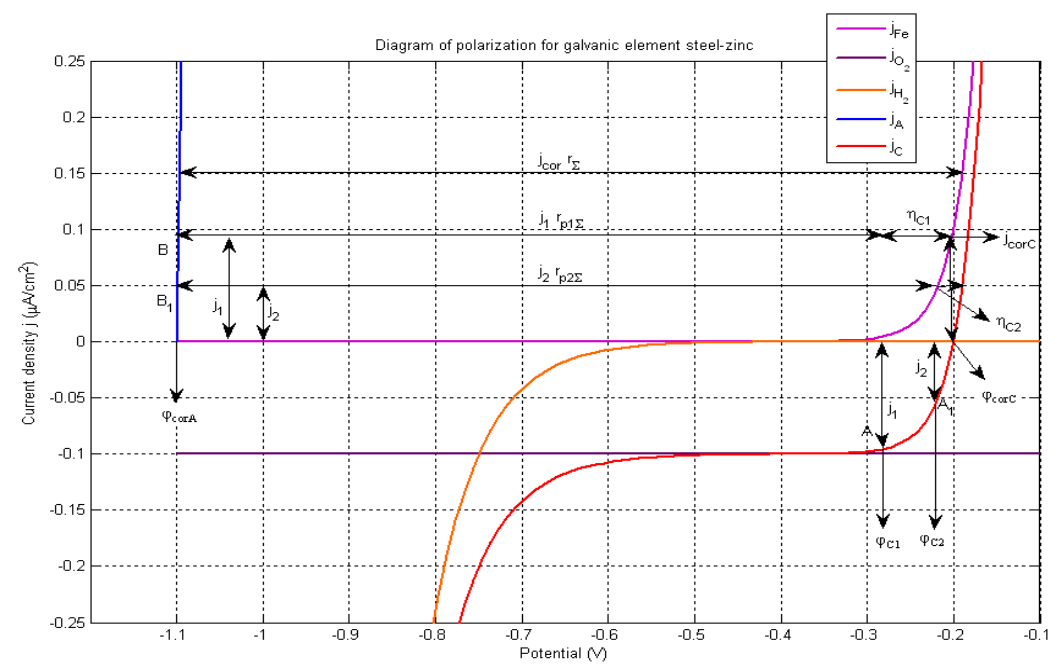

Figure 4: Polarization diagram of galvanic element steel/zinc (vs. $\left.\mathrm{Cu} / \mathrm{CuSO}_{4}\right)$.

\subsubsection{Indirect boundary element method}

When analyzing CP systems, finding values of the potential and protection current density on the boundary surface is of particular interest. Therefore, the boundary element method is an ideal method for solving this problem. The current field of the CP system, for a single or double-layer soil, is described by Fredholm's integral equation of the first kind:

$$
F(P, Q)=\rho_{U} \int_{S} j(P) \cdot G(P, Q)-\varphi_{0}(Q)=0,
$$

where: $P$ - field source point,

$Q$ - observation point,

$j(P)$ - surface current density,

$G(P, Q)$ - Green's function,

$\varphi_{0}(Q)$ - a function of potential at observation point,

$\rho_{U}-$ soil resistivity of upper layer,

$S$ - surface.

The corresponding Green's functions are:

- For a homogeneous soil:

$$
G(P, Q)=\frac{1}{4 \pi}\left(\frac{1}{r_{P 1, Q}}+\frac{1}{r_{P 2, Q}}\right),
$$


- For double-layer soil, in the case when the field source is located in the upper layer:

$G(P, Q)=\frac{1}{4 \pi}\left[\frac{1}{r_{P 1, Q}}+\frac{1}{r_{P 2, Q}}+\sum_{n=1}^{\infty} \beta^{n}\left(\frac{1}{r_{P 3, Q}^{n}}+\frac{1}{r_{P 4, Q}^{n}}+\frac{1}{r_{P 5, Q}^{n}}+\frac{1}{r_{P 6, Q}^{n}}\right)\right]$,

where: $r_{P, Q}$ is the distance between the field source point and observation points.

Solving Fredholm's integral equations of the first kind is done using the indirect boundary element method. Boundary surfaces are discretized using 2D biquadratic boundary elements. The Collocation method is applied at the point, so that the weight function is a Dirac delta function. Applying the Collocation method at the point, an integral expression for a boundary element can be written as:

$$
\begin{gathered}
\sum_{e=1}^{n_{e}} \int_{S^{e}} \delta\left(Q-Q_{i}\right) \cdot F(P, Q) \cdot d S^{e}=\sum_{e=1}^{n_{e}} \rho_{U} \cdot \int_{-1}^{+1} \int_{-1}^{+1} \sum_{i=1}^{9} N_{i}\left(\xi_{P}, \eta_{P}\right) \cdot j_{i} \\
\cdot \operatorname{det}\left[J\left(\xi_{P}, \eta_{P}\right)\right] \cdot G\left(\xi_{m_{1}}, \eta_{m_{2}} ; Q\right) \cdot d \xi \cdot d \eta-\varphi_{0}\left(Q_{i}\right)=0
\end{gathered}
$$

After the application of Gauss-Legendre's quadrature formulas on eqn. (12) it can be written as:

$$
\begin{gathered}
\sum_{e=1}^{n_{e}} \sum_{m_{1}=1}^{n_{G}} \sum_{m_{2}=1}^{n_{G}} \sum_{i=1}^{9} N_{i}\left(\xi_{m_{1}}, \eta_{m 2}\right) \cdot j_{i} \cdot G\left(\xi_{m_{1}}, \eta_{m_{2}} ; Q_{i}\right) \\
\cdot \operatorname{det}\left[J\left(\xi_{m_{1}}, \eta_{m_{2}}\right)\right] \cdot w_{m_{1}} \cdot w_{m_{2}}-\varphi_{0}\left(Q_{i}\right)=0
\end{gathered}
$$

where: $N(\xi, \eta)$ - shape function,

$\xi, \eta$ - Gauss points,

$w_{i}$ and $w_{j}$ - weights coefficients for Gauss points $\xi$ and $\eta$, respectively.

In order to solve this system of equations, it is necessary to add another system of equations, as follows:

$$
\begin{aligned}
& \sum_{e=1}^{n_{e}} \int_{S} j \cdot d S=\sum_{e=1}^{n_{e}} \rho_{U} \int_{-1}^{+1} \int_{-1}^{+1} \sum_{i=1}^{9} N_{i}\left(\xi_{P}, \eta_{P}\right) \cdot j_{i} \cdot \operatorname{det}\left[J\left(\xi_{P}, \eta_{P}\right)\right] \cdot d \xi \cdot d \eta \\
& \sum_{e=1}^{n_{e}} \sum_{m_{1}=1}^{n_{G}} \sum_{m_{2}}^{n_{G}} \sum_{i=1}^{9} N_{i}\left(\xi_{m_{1}}, \eta_{m_{2}}\right) \cdot j_{i} \cdot \operatorname{det}\left[J\left(\xi_{m_{1}}, \eta_{m_{2}}\right)\right] \cdot w_{m_{1}} \cdot w_{m_{2}}=0
\end{aligned}
$$

Previously written systems of eqns. (13) and (15) can be expressed in matrix form:

$$
\left[\begin{array}{ll}
h_{a a} & h_{a c} \\
h_{c a} & h_{c c}
\end{array}\right] \cdot\left\{\begin{array}{l}
\varphi_{a} \\
\varphi_{c}
\end{array}\right\}=\left[\begin{array}{ll}
g_{a a} & g_{a c} \\
g_{c a} & g_{c c}
\end{array}\right] \cdot\left\{\begin{array}{c}
j_{a} \\
j_{c}
\end{array}\right\}
$$

where: $[\boldsymbol{h}]$ and $[\boldsymbol{g}]$ - matrix of coefficients,

$\{\boldsymbol{\varphi}\}$ - column vector of potential,

$\{j\}$ - column vector of current density. 
By solving this system of eqn. (16), values of the protection current density and potential can be obtained.

Figure 5 shows the distribution of the protective potential of the CP system with galvanic anodes of the non-isolated steel pipeline, for homogeneous soil with soil resistivity $\rho_{U}=50 \Omega \mathrm{m}$. Figure 6 shows the same CP system in doublelayer soil with a soil resistivity of the upper layer $\rho_{U}=50 \Omega \mathrm{m}$, thickness of the upper layer $h=10$ and soil resistivity of the lower layer $\rho_{L}=1000 \Omega \mathrm{m}$. The pipeline has a diameter of $4 \mathrm{~m}$, and it is buried at a depth of $3.75 \mathrm{~m}$. Anode strings are placed on both sides of the pipeline at a distance of $5.5 \mathrm{~m}$ away from the pipeline axis. The burial depth of the anode strings is $2.8 \mathrm{~m}$, and its length is $6 \mathrm{~m}$.

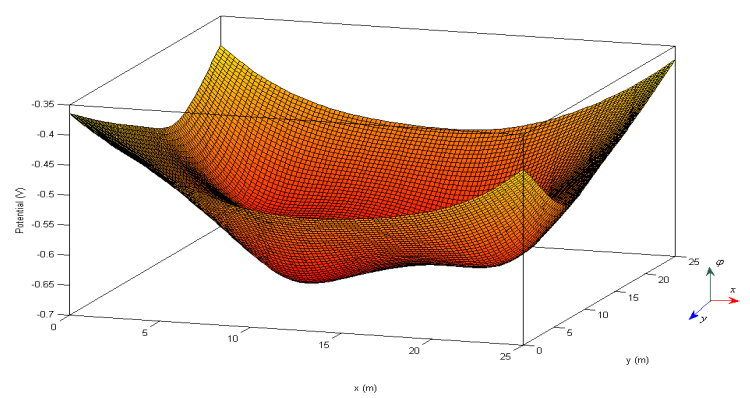

Figure 5: $\quad$ Potential distribution of CP system in single-layer soil.

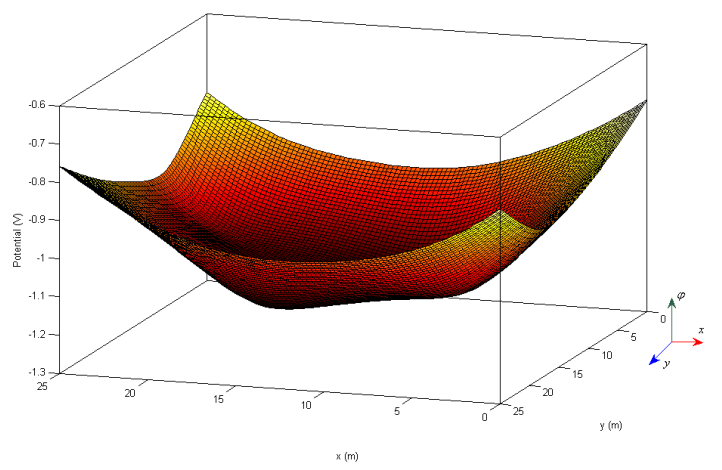

Figure 6: Potential distribution of CP system in double-layer soil.

Figure 7 gives comparison of protective potential values for single-layer and double-layer soil. It can be noticed that the absolute value of the protective potential increases as the electrical resistivity of the lower layer increases. In such situations, neglecting the multiple layers of soil leads to overprotection of a protected object and high economic expenses. 


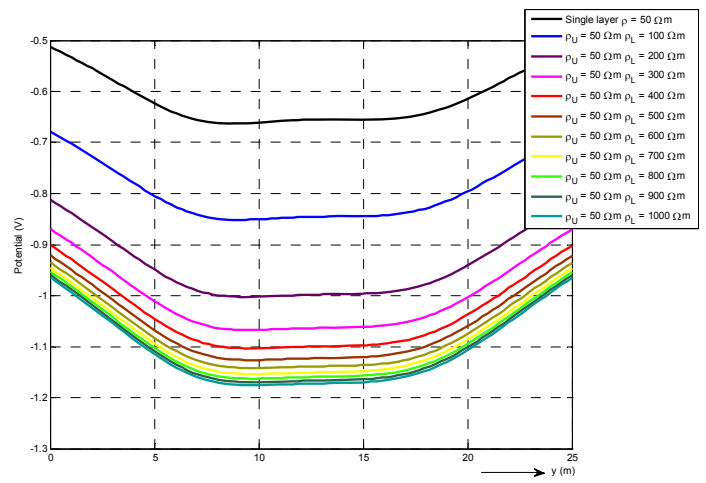

Figure 7: Comparison of the equipotential lines of the CP system for cases of single-layer and double-layer soil for $\mathrm{x}=12.5 \mathrm{~m} ; 0 \mathrm{~m}<\mathrm{y}<25 \mathrm{~m}$.

Figure 8.a gives the value of the percentage error of the potential, in the case of neglecting multiple layers of soil. It is noticeable that with the increase of soil resistivity, the percentage systematic error also increases, over $40 \%$. In these situations, from a technical point of view, neglecting multiple layers has a positive effect, because an object becomes overprotected. On the other hand, from an economic point of view, the cost of equipment increases. Therefore, for the large differences in the values of soil resistivity, between neighboring layers, it is necessary to take into account the multiple layers of electrolyte. Figure 8.b shows how the percentage systematic error changes with the change of thickness of the upper layer (the layer in which protected object and anode strings are placed). We considered double-layer soil whose upper layer has variable thickness and soil resistivity $\rho_{U}=50 \Omega \mathrm{m}$, and the soil resistivity of the lower layer is $\rho_{L}=1000 \Omega \mathrm{m}$ in the first case, and $\rho_{L}=500 \Omega \mathrm{m}$ in the second case. In both situations it is noticeable that error decreases with increasing thickness of the upper layer of soil.

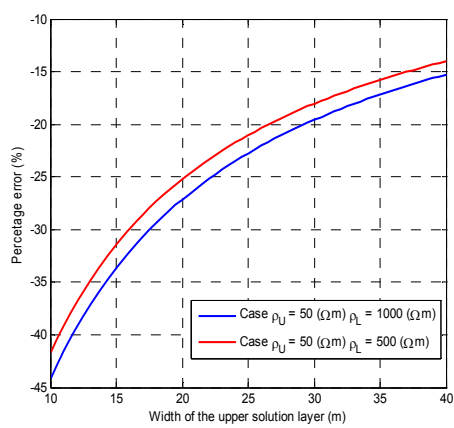

a)

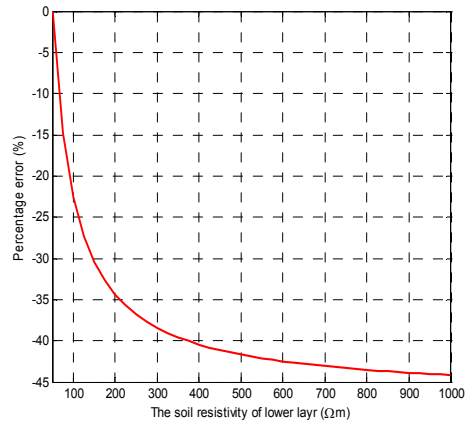

b)

Figure 8: $\quad$ Percentage errors in the calculation of potential of the CP system. 


\section{Conclusion}

The main task in determining the optimal CP system with galvanic anodes is to define technically the correct distribution of the protective current density and potential. Usually, the static current field of the CP system is calculated by solving the integral field equation. When applying the CP system to underground steel structures, geometries as well as nonlinear mathematical interpretation of electrochemical reactions are very complex. Therefore, the integral field equation can only be solved using numerical techniques. Also, considering the fact that the electrolyte is inhomogeneous, the paper analyzes the effect of discontinuity of electrolyte conductivity on the distribution of the protective potential of the CP system with galvanic anodes. We have solved this problem by using the indirect boundary element method. Paper also gives analysis of systematic errors that appear when neglecting the fact that electrolyte is composed of more layers. It turned out that the errors in the protective potential calculation can happen and they can exceed $40 \%$. Also, in a case of double-layer soil with large thickness of upper layer, soil can be modeled as single-layered. In this case, soil resistivity of a single-layer is equal to a soil resistivity of upper layer in a double-layer model. This conclusion follows from the fact that the percentage systematic error decreases with increasing thickness of the upper layer.

\section{References}

[1] D. P. Riemer and M. E. Orazem, Modelling coating flaws with nonlinear polarization curves for long pipelines, Advices in Boundary Elements, Voll. 12, pp 225-259, Sauthampton, WIT Pres, 2006.

[2] D. P. Riemer, Modelling Cathodic Protection for pipeline networks, $\mathrm{Ph}$. D. Dissertation, University of Florida, Gainesville, FL, 2000.

[3] R. A. Adey, C. A. Brebbia and S. M. Niku, Application of boundary element method in corrosion engineering, Topics in Boundary Element Research, Computational Mechanich Publications, 1990.

[4] A. B. Peratta, J. M. W. Baynham and R. A. Adey, Computational modelling of cathodic protection systems for pipelines in multi-layer soil, WIT Transactions on Engineering Sciences, Vol 65, (c) 2009 WIT Press.

[5] T. Barić, V. Boras, S. Nikolovski, Analysing the effect of discontinuity of electrical conductivity of soil on ground resistance, Energija Journal of Energy, Voll 55 No 4, pp 454-473, Zagreb 2006.

[6] Z. Haznadar, Z. Stih, Electromagnetic fields, waves and numerical methods, IOS Press, Amsterdam, 2000.

[7] W. Baeckmann, W. Schwenk and W. Prinz, Handbook of Cathodic Corrosion Protection, Gulf Publishing Company, Houston, Texas, 1997.

[8] A. Muharemovic, I. Turkovic, A. Muharemovic, S. Tasakovic, A. Mujezinovic, Calculation methods of cathodic protection system parameters with vertical anode zinc string, $20^{\mathrm{TH}}$ International expert meeting, Power engineering, Maribor, Slovenia, 2011. 
[9] A. Muharemovic, H. Zildzo and E. Letic, Modelling of protective potentials distribution in cathodic protection systems using coupled BEM/FEM method, $30^{\mathrm{TH}}$ International conference on Boundary Elements Method and Other Reduction Methods, BEM/MRM 30, Maribor, Slovenia, 2008. 